\title{
Prototype System for Marking and Identification of Dangerous Spots for Vision Impaired People - Size and Shape Testing Marking the Dangerous Spots Zone
}

\author{
J. WiCIAK ${ }^{a *}$, B. Borkowski ${ }^{a}$ AND I. CzAJKA ${ }^{b}$ \\ ${ }^{a} \mathrm{AGH}$ - University of Science and Technology, Faculty of Mechanical Engineering and Robotics \\ Department of Mechanics and Vibroacoustics, al. A. Mickiewicza 30, 30-059 Krakow, Poland \\ ${ }^{b} \mathrm{AGH}$ - University of Science and Technology, Faculty of Mechanical Engineering and Robotics \\ Department of Power Systems and Environmental Protection Facilities, al. A. Mickiewicza 30, 30-059 Krakow \\ The article constitutes the continuation of the works realized under the research project entitled: "The \\ development of the method for marking and identification of the spots dangerous and of special importance for \\ vision impaired persons in the big city with use of the wave-vibration markers". The consideration included the \\ identification of the object (human), identification of the direction of movement (double barrier), wireless data \\ transmission with the information on the dangerous area, stimulation of the vibration signal and the feedback \\ of the system. This article presents the result of choice of the optimal solution regarding the installation of the \\ system on the selected objects of the urban engineering and also presents the concept of the method of modelling \\ of the shape of the zone that is used for marking of the dangerous spot.
}

PACS: 41.20.-q, 46.40.-f, 07.55.Db, 07.55.Ge

\section{Introduction}

The current methods of marking of the dangerous spots - the sound signalling, special pavement surface etc. - are sufficient in the stationary objects such as the pedestrian crossing, stairways or the platforms, but are not flaw-free [1]. For example the snow might effectively cover the pavement surface making it totally inefficient in marking the dangerous area. Also the sound signalling might be a problem, by being a noise source (the disturbing sound in the area of pedestrian crossing, especially at the night), also the lack of the uniformity of sound might be a problem - there may be several types of sound in one urban area. Finding the traffic light activation button usually is a big problem. The additional problem is marking of the object that exists only in limited time on the given route, e.g. the road works, excavation or the scaffolding. That is why authors decided to develop the system for direct warning and information.

Since 2009 the research project entitled: "The development of the method for marking and identification of the spots dangerous and of special importance for vision impaired persons in the big city with use of the wave-vibration markers" has been realized and the prototype version of the transmitter and the receiver is being developed $[2,4]$. At the first stage of the project - the survey held among the people with vision impairment about the

\footnotetext{
* corresponding author; e-mail: wiciak@agh.edu.pl
}

sound perception from the environment and related problems are realized $[2,3]$. The obtained results say that if there are too many sounds which disturb each other, they may be incorrectly interpreted [3]. The selection of the method of data transmission with the use of vibration is very accurate, since about $70 \%$ of respondents stated that the vibration method will be helpful in commuting.

The solutions available in the market which have been subjected to the assessment are presented in the monograph [3]. Results are gathered in the table with surprising fact that the intermediate solutions might bring a lot of problems, e.g. [2]. In the article [4] the concept of the system for identification of the spots dangerous for vision impaired persons in the large urban area was presented. Also the concept of utilization of the alternative wireless communication system was presented, that is usage of the infrared, Bluetooth and RFID [4-6].

This article presents the result of choice of the optimal solution regarding the installation of the system on the selected objects of the urban engineering and also presents the concept of the method of modelling of the shape of the zone that is used for marking of dangerous spot.

\section{The concept of the system}

The aim of the project is the development of the transmission-reception method for identification of the dangerous and specially important areas. 
The necessary stage of the project realisation is also the development of the method for teaching of the vision impaired persons (youth and children) of the identification of the dangerous spots in the urban area based on the wave-vibration markers.

The authors of the project focused first on the dangerous spots such as:

— stairway (separately up/down directions),

- railway platform,

- pedestrians crossing,

- all types of areas/obstacles existing only in given time, e.g. excavations, road works, bridges.

The system also needs to be universal, easy for installation, where the deciding factor is its introduction and maintenance economy.

The requirements stated in [3] are:

- definition of "coarse" localisation (first signal on approach to the dangerous zone),

- fine definition of the localisation, needed much more accuracy of localisation of the person (second information about the dangerous zone),

- signalling with vibration.

Figure 1 presents the main idea and assumptions for the system. By following the above assumptions the prototype of the transmission system and reception system as a bracelet is developed.

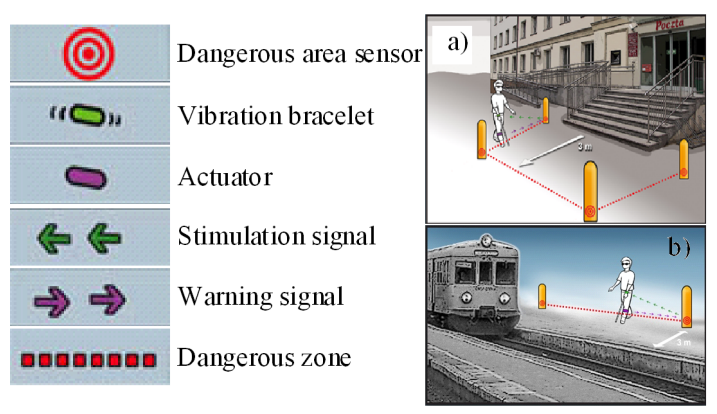

Fig. 1. (a) Dangerous zone stairway, (b) dangerous zone platform.

The transmission system is located on the whole area marked as the dangerous zone, and the reception system is given to the user. Also reception system is an individual identifier for each user and that is necessary if two or more people equipped with the system appear in the dangerous zone. The system is activated (the bracelet sends the signal) when the person is found in the region of the dangerous zone (determined by a mat, as in Fig. 2), after the activation the transmission module sends the encoded signal to the bracelet, with information about the type of the danger.

The bracelet will have vibration motors built-in (similar to the cell-phones), and with adequate sequence it will give the information.

Figure 3 shows the prototype of the bracelet, which is meant to activate the system and inform about the type of danger. The prototype consists of the transmission/

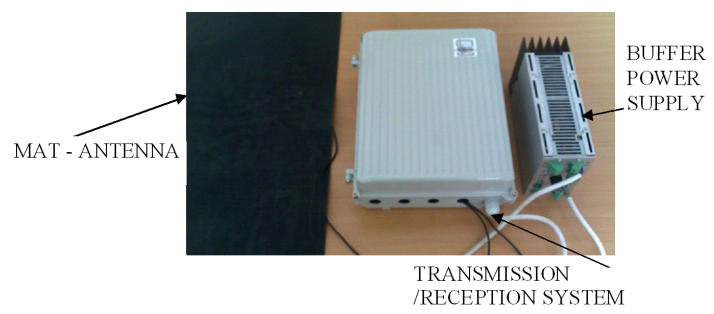

Fig. 2. Transmission/reception system.

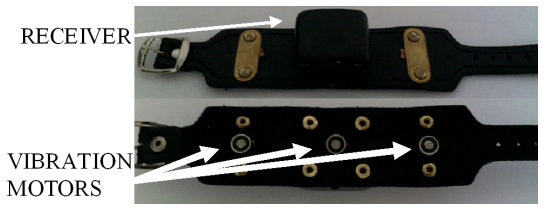

Fig. 3. Bracelet.

reception system locked in the black cube and the leather belt with mounted three vibrator motors. Through the metal parts on both ends the accumulator is charged. The identifier is individual for each user since in general case more than one person might be in the region of the dangerous zone.

\section{Testing size and shape of marking zone of the dangerous spot}

Marking of the dangerous zone needs shaping of the electromagnetic field generated by the described system in such a manner to gain all the information about the zone in shortest distance possible. To achieve that goal the authors use numeric methods of modelling of the electromagnetic field.

The equations defining the electromagnetic field are known for long time. The magnetic field is defined by the Ampere law. In situation when the field is subjected to slow variations in time, a stationary model could be used. When there are no ferromagnetisms, the magnetostatics problem may be expressed with magnetic vector potential $\boldsymbol{A}$. Magnetic vector potential satisfies equation $\boldsymbol{B}=\nabla \times \boldsymbol{A}$, which is obtained from the Ampere law, with displacement current ignored, that (1):

$$
\nabla \times\left(\frac{1}{\mu} \nabla \times \boldsymbol{A}\right)=\boldsymbol{j},
$$

where $\mu$ is the magnetic permeability of the material and $j$ is the current density [7]. Earlier mentioned equation may be solved in three dimensions, a curl vector identity is used to transform the equation into the form (2):

$$
\frac{-1}{\mu} \nabla^{2} \boldsymbol{A}=\boldsymbol{j},
$$

which is valid when $\mu$ is not a function of space coordinates. In time harmonic case the current density is sinusoidal function of time $\boldsymbol{j}=\boldsymbol{j}_{0} \mathrm{e}^{\mathrm{i} \omega t}$. 
The term of slow variation is an imaginary term, but in the analysed case the frequency of the system is $50 \mathrm{kHz}$, which means that the wavelength is about 6000 meters. Thus the wavelength is fairly long in comparison to the transmission system. The calculations were performed with finite element method using the Elmer package [8]. The virtue of the presented software is that it is an open software, which, from the scientist's point of view is very profitable. The model was built with use of the gmsh software [9], in which the net of finite elements was applied with the parabolic shape functions. The 3D model of size 10 to $10 \mathrm{~m}$ tall and 5 meters was analyzed. The study area had the shape of the hemisphere. The results obtained in the form of the magnetic vector potential in the selected plane perpendicular to the direction of the radiator pipes are presented in Figs. 4-7. The impact of wires that can find near the transmitting antenna was not taken into account.

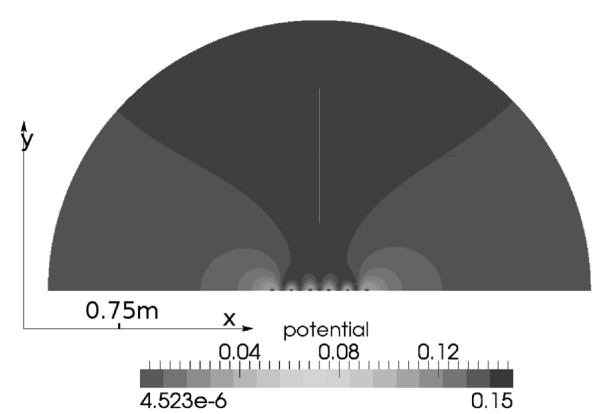

Fig. 4. Serial system, large distance between the conductors.

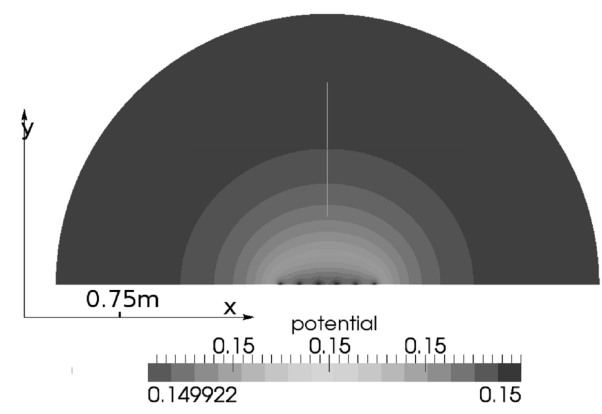

Fig. 5. Parallel system, large distance between the conductors.

Since the research is only in the preliminary stage, the partial results will be presented. The described results relate to two systems of conductors presented in Fig. 8. In reality the conductors are laid out on the rubber mat. The numeric model does not consider the influence of the mat and the base on the distribution of the electromagnetic field.

The system (a) was called the serial system and the system (b) was called the parallel system. In both cases the density of the current was assumed to be the same,

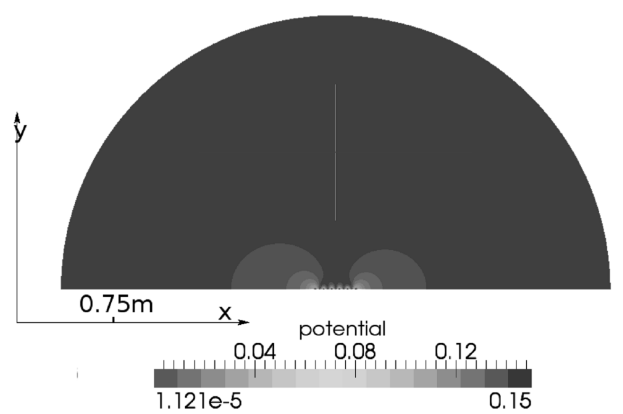

Fig. 6. Serial system, small distance between the conductors.

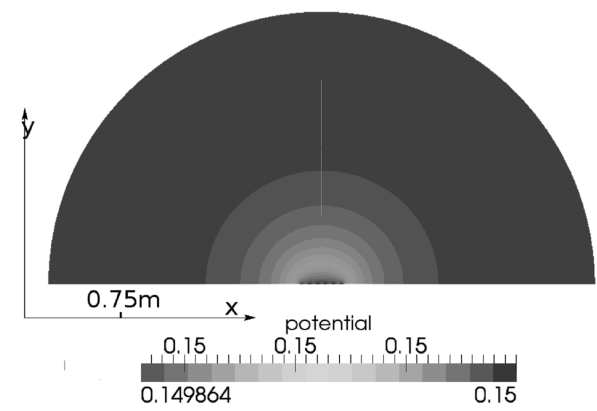

Fig. 7. Parallel system, small distance between the conductors.

Fig. 8.

The systems were tested with different distances between the conductors and different currents in the conductors. The selected results were presented in Figs. 4-7. Colours show magnetic vector potential amplitude in $\mathrm{V} \mathrm{s} \mathrm{m}^{-1}$. Figures 4 and 5 show distribution of the magnetic field for, respectively, serial and parallel system with large distances. Figures 6 and 7 show distribution of magnetic field with small distances between conductors.

Large distance between the conductors means $0.3 \mathrm{~m}$, and small distance means $0.05 \mathrm{~m}$. Figures $4-7$ clearly show that irrespective of the distance the serial system allows more precise marking of the dangerous zone than the parallel system. Still the values of the intensity of the electromagnetic field are significantly lower. Also the physical problems occur related to the phenomena of the

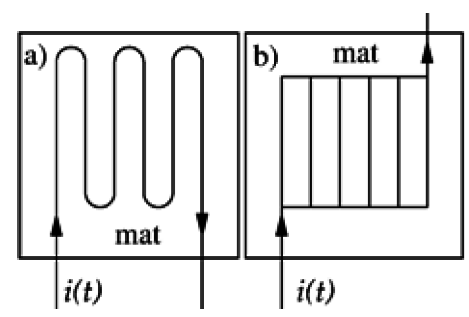

Fig. 8. The analysed layouts of the conductors. 
emission of the electromagnetic waves. To achieve narrow beam of waves it is necessary to use the radiator of dimension much bigger than the wavelength, which is not possible with the selected working frequency. For that reason it is necessary to concentrate on such wave phenomena as the interference which allows creation of strong local maximum of the electromagnetic field.

\section{Conclusions}

Since the beginning of the project there were three versions of the project developed and only the last one fulfils all the assumed requirements. The marking of the dangerous zone might be of unlimited shape and size. Nevertheless in front of the authors there are many application tests, reliability tests, and simulation of the shape of the field. Also there will be more research on combining the system with the systems for controlling of the traffic lights and controlling of the information system of transportation company. An additional virtue of the system is the fact that it will be useful also for the tourist information systems.

\section{Acknowledgments}

The work was conducted in the framework of the development project NR17001706 financed by the National Centre for Research and Development (NCBiR).

The authors wish to thank the Department of the $\mathrm{Mu}-$ nicipal Infrastructure and Transportation in Kraków for providing the traffic light equipment for research and testing.

\section{References}

[1] M. Wysocki, Environmental Design for People with Disabilities, Gdańsk University of Technology, Gdańsk 2010

[2] J. Wiciak, D. Młynarczyk, B. Borkowski, Medical News 79, 3 (2010).

[3] J. Wiciak, D. Młynarczyk, in: Rehabilitation of People with Dysfunctions Sense of Hearing and Sight, Eds. D. Hojan-Jezierska, F. Jaroszyk, L. Kubisz, Monograph Stanisław Staszic Higher Vocational State School, Piła 2011.

[4] B. Borkowski, J. Wiciak, Acta Phys. Pol. A $\mathbf{1 1 9}$, 921-A (2011).

[5] B. Czechyra, E. Skrodzka, G. Szymanski, F. Tomaszewski, J. Wiciak, Pol. J. Environm. Studies 20, 44 (2011).

[6] http://rfid-lab.pl/.

[7] P. Russer, Electromagnetics, Microwave Circuit and Antenna Design for Communications Engineering, Artech House, London 2006.

[8] http://www.csc.fi/english/pages/elmer .

[9] C. Geuzaine, J.-F. Remacle, Int. J. Num. Meth. Eng. 79, 1309 (2009). 